\title{
Kopf-Hals-Tumoren: Überlebensvorteil für Cetuximab-Kombination
}

- Patienten mit Plattenepithelkarzinom im Kopf-Hals Bereich (SCCHN) im rezidivierten und/oder metastasierten Stadium profitieren sowohl hinsichtlich des Gesamtüberlebens als auch des progressionsfreien Überlebens wenn zu einer platinbasierten Chemotherapie der monoklonale Antikörper Cetuximab (Erbitux ${ }^{\circledR}$ ) gegeben wird.

Zu diesem Ergebnis kommt die EXTREMEStudie [Vermorken JB et al. N Engl Med, 2008; 359: 1116-1127]. Wie PD Dr. Ulrich Keller, München, anhand der Studienergebnisse zeigen konnte, verbesserte sich das Gesamtüberleben signifikant von 7,7 Monate auf 10,1 Monate. „Beim progressionsfreien Überleben kam es zu einer Verbesserung von 3,3 auf 5,6 Monate", so der Experte weiter. Da die Therapie jedoch sehr aggressiv sei, wird sie laut Leitlinie nur für Patienten mit gutem Allgemeinzustand als Erstlinientherapie empfohlen.

In der sogenannten Bronner-Studie [Bronner JA et al. N Engl J Med, 2006; 354:567578] wurde die Wirksamkeit einer Strahlentherapie in Vergleich zu Strahlentherapie in Kombination mit Cetuximab bei Patienten mit unbehandelten lokal fortgeschrittenen SCCHN untersucht. „Aufgrund der Bronner-Daten hat der Anteil der Therapie mit Cetuximab deutlich zugenommen“, sagte Prof. Dr. Jürgen Debus, Heidelberg. Denn es konnte gezeigt werden, dass die Kombinationstherapie die lokoregionale Kontrolle hochsignifikant verbessert. Die aktuell erschienen Daten des 5-Jahres-Update der Studie [Bronner JA et al. Lancet Oncol, 2010; 11 (1): 21-8] zeigen einen an- haltenden Überlebensvorteil von rund 9,2 Prozent. „Die Lebensqualität unterschied sich in den beiden Armen nicht", erläuterte Debus. Die häufigste Nebenwirkung unter der Cetuximab-Kombination sei ein akneartiger Hautausschlag, dessen Schweregrad signifikant mit der Verbesserung des Gesamtüberlebens korreliert. Inwieweit die auftretenden Hautreaktionen einen prädiktiven Stellenwert haben, wird, so Debus, zur Zeit in einer multizentrischen Phase-IV-Studie untersucht.

cvs

Satellitensymposium: „Kopf-Hals-Tumore: Eine interdisziplinäre Herausforderung Therapiestandards und neue Perspektiven“, Berlin, Oktober 2010, Veranstalter: Merck Serono

\section{Mit pflanzlichem Sekretolytikum gegen Atemwegserkrankungen}

— Die akute Form einer Sinusitis wird meist durch den Verschluss des Nebenhöhlen-Ostiums verursacht. Als Folge, so Prof. Dr. Hans Behrbohm, Berlin, kommt es zu Störungen von Ventilation und Drainage sowie zur Sekretstagnation. Im weiteren Verlauf ändert sich das Milieu der Nasennebenhöhle mit Begünstigung von Keimwachstum und Entzündungsreaktion. Typische Symptome sind nasale Obstruktion, Kopf- und Gesichtsschmerz (insbesondere beim Bücken), Hyposmie und nasale Sekretion. Bei chronischer Rhinosinusitis und Polyposis nasi sind als Ursachen immunologische Disposition, ein verändertes Spektrum von Entzündungszellen in den Nebenhöhlen, Pilze und Staphylokokken-Superantigene sowie eine ASS-Intoleranz von Bedeutung.

Im Rahmen chronisch entzündlicher Schleimhauterkrankungen kommt es zu Veränderungen des Mukoziliarapparates: Sekretorische Becherzellen nehmen ab, die Drüsen bilden vermehrt mukösen anstatt serösen Schleim und der Zilienapparat degeneriert. Bei einer Sinusitis kann es zum sogenannten Etagenwechsel mit Beteiligung der Bronchien kommen. Die Atemwege sind dabei pathophysiologisch als Einheit - „United Airways“ - zu verstehen.
Auf die Bedeutung von Myrtol standardisiert (GeloMyrtol ${ }^{\circledR}$ ) bei Sinusitis und Bronchitis wies Dr. Thorsten Zehlicke, Hamburg, hin. Dieses Präparat mit seinen ätherischen Ölen wirkt in erster Linie sekretolytisch, sekretomotorisch und mukolytisch. Zusätzlich sind auch antimikrobiell-antiinflammatorische, antioxidative und bonchospasmolytische Effekte bekannt.

Die Wirkungen von Myrtol standardisiert sind in zahlreichen präklinisch-experimentellen und klinischen Studien seit längerem dokumentiert. Zehlicke machte auf eine Good-Clinical-Practice-konforme randomisiert-kontrollierte Studie aufmerksam, in der 331 Sinusitis-Patienten mit dem MyrtolPräparat oder Placebo behandelt wurden. Im Therapieverlauf war eine signifikante Verbesserung vor allem der Kernsymptome Kopfschmerz, Schmerz beim Bücken und Druckschmerz über den Nervenaustrittspunkten nachweisbar (Federspil P et al.Laryngo-Rhino-Otol 1997; 76: 23-7). In einer anderen Studie wurden 676 Bronchitis-Patienten entweder mit Myrtol standardisiert, Cefuroxim, Ambroxol oder Placebo therapiert. Das Myrtolpräparat zeigte nach einer Woche mit 95\% die höchste Ansprechrate und war - mit Vorteilen in einigen klinischen Parametern - vergleichbar

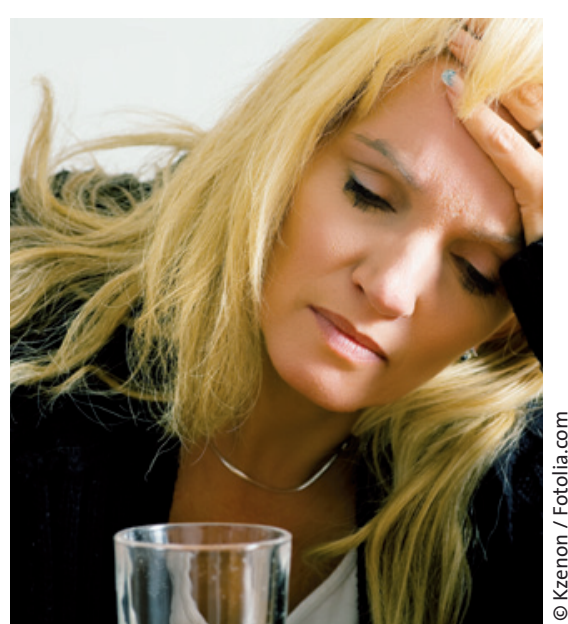

Typische Symptome der Sinusitis: Kopfschmerz vor allem beim Bücken und Druckschmerz an den Nervenaustrittspunkten

effektiv wie die anderen Verumsubstanzen (Matthys $\mathrm{H}$ et al. Arzneim-Forsch./Drug Res 2000; 50/8: 700-11). Dr. Andreas Fischer

Pressekonferenz „State of the art der Therapie der Rhinosinusitis " anlässlich der EXPOPHARM, München, 8. Oktober 2010; Veranstalter: Pohl-Boskamp 